UNIVERSIDADE DE SÃO PAULO

INSTITUTO DE PSICOLOGIA

GLAUSA DE OLIVEIRA MUNDURUCA

Contribuição para o estudo da constituição psíquica de mulheres alcoolistas 
GLAUSA DE OLIVEIRA MUNDURUCA

\section{Contribuição para o estudo da constituição psíquica de mulheres alcoolistas}

Tese apresentada ao Instituto de Psicologia da Universidade de São Paulo, para obtenção do título de Doutor em Psicologia.

Área de concentração: Psicologia Escolar e do Desenvolvimento Humano.

Orientadora: Prof ${ }^{a} \operatorname{Dr}^{\mathrm{a}}$ Audrey Setton Lopes de Souza 
AUTORIZO A REPRODUÇÃO E DIVULGAÇÃO TOTAL OU PARCIAL DESTE TRABALHO, POR QUALQUER MEIO CONVENCIONAL OU ELETRÔNICO, PARA FINS DE ESTUDO E PESQUISA, DESDE QUE CITADA A FONTE.

Catalogação na publicação

Serviço de Biblioteca e Documentação

Instituto de Psicologia da Universidade de São Paulo

Munduruca, Glausa de Oliveira.

Contribuição para o estudo da constituição psíquica de mulheres alcoolistas / Glausa de Oliveira Munduruca; orientadora Audrey Setton Lopes de Souza. -- São Paulo, 2008.

$204 \mathrm{f}$.

Tese (Doutorado - Programa de Pós-Graduação em Psicologia. Área de Concentração: Psicologia Escolar e do Desenvolvimento Humano) - Instituto de Psicologia da Universidade de São Paulo.

1. Alcoolismo 2. Mulheres 3. Constituição do sujeito 4. Psicodiagnóstico 5. Psicanálise 6.Teste de Rorschach I. Título. 


\section{FOLHA DE APROVAÇÃO}

Glausa de Oliveira Munduruca

Contribuição para o estudo da constituição psíquica de mulheres alcoolistas

Tese apresentada ao Instituto de Psicologia da Universidade de São Paulo, para obtenção do título de Doutor em Psicologia.

Área de concentração: Psicologia Escolar e do Desenvolvimento Humano.

Aprovado em:

Banca Examinadora

Prof. Dr. Arthur Guerra de Andrade

Instituição: FMUSP - IPq Assinatura:

$\operatorname{Prof}^{a} \operatorname{Dr}^{\mathrm{a}}$ Monica Levit Zilberman

Instituição: FMUSP - IPq Assinatura:

Prof. Dr. Andrés Eduardo Aguierre Antúnez

Instituição: IPUSP

Assinatura:

Prof. Dr. Christian Ingo Lenz Dunker

Instituição: IPUSP

Assinatura:

$\operatorname{Prof}^{a} \operatorname{Dr}^{\mathrm{a}}$ Audrey Setton Lopes de Souza

Instituição: IPUSP

Assinatura: 


\section{AGRADECIMENTOS}

A minha orientadora, $\operatorname{Prof}^{\mathrm{a}} \operatorname{Dr}^{\mathrm{a}}$ Audrey Setton Lopes de Souza, por respeitar meu tempo e meu modo de pensar e por ter sido capaz de iluminar a forma de apresentação dos resultados, quando eu já não acreditava mais.

A meu mestre, Dr. Roberto Azevedo, símbolo de sabedoria e capacidade de transmissão, por tudo o que me ensinou, de modo incansável e apaixonado.

Ao Prof. Dr. Arthur Guerra de Andrade, pelo exemplo da possibilidade de integração de diferentes saberes e por ter me ajudado generosamente desde o início desta pesquisa.

À $\operatorname{Prof}^{\mathrm{a}} \operatorname{Dr}^{\mathrm{a}}$ Monica Zilberman, que ajudou a abrir caminhos para o estudo da dependência de substâncias em mulheres, sendo o seu trabalho fonte inspiradora para o meu.

Ao Prof. Dr. Christian Dunker, por ter fornecido os elementos fundamentais para a reorganização do meu texto durante o Exame de Qualificação.

Ao Prof. Dr. Andrés Antúnez, por sua disposição em colaborar.

Ao Dr. Jorge Figueiredo, por confiar em mim desde o princípio e contribuir concretamente para a realização desta pesquisa.

A Giselle Petri Costa, rorschachista brilhante, que me acompanhou passo a passo nesta pesquisa.

A Marizilda Paulino, minha grande interlocutora.

Ao Dr. Durval Mazzei Nogueira Filho, pela discussão teórica e pela supervisão de algumas entrevistas. 
A Tatiana Melhado, doutoranda em Estatística do Instituto de Matemática e Estatística da USP, por fornecer os pilares da análise estatística desta pesquisa.

A João Antonio Piazoto e Maria Nozoseletki, pela tradução do artigo russo.

À equipe da Confraria de Textos, pela revisão do texto no tempo exato.

A minha analista, Angelina Harari, por saber me conduzir no emaranhado de idéias que surgiram em minha mente.

A Christiane Prado, a primeira a estender a mão, por compartilhar as minhas angústias e suportar comigo o desejo por bebês no plano simbólico.

A Luciano Chati, por me indicar o caminho.

A Terezinha Romão, por me acolher.

A Thais Rodrigues e Elaine Simões, psicólogas da Clínica de Psiquiatria do HSPM, por me ensinarem a trabalhar em grupos terapêuticos.

A meus queridos amigos do grupo de supervisão, Besita Suplicy, Cibele Rosa, Juliana Braga, Lygia Fabbro, Ricardo Pizza e Silvia Milnitzy, por suas inúmeras contribuições.

A Marcos Nakashima, por me socorrer num dos momentos mais difíceis da minha vida.

A meu irmão, Gláucio Munduruca, que, partilhando seu conhecimento e sua sensibilidade musical, muito me tem ajudado na minha escrita. Uma nota pede a outra...

Às mulheres que participaram desta pesquisa, permitindo a minha presença. 


\section{RESUMO}

MUNDURUCA, G. O. Contribuição para o estudo da constituição psíquica de mulheres alcoolistas. 2008, 204 f. Tese (Doutorado) - Instituto de Psicologia, Universidade de São Paulo, São Paulo, 2008.

Este estudo investiga a constituição psíquica de mulheres alcoolistas, procurando ampliar a compreensão de seu modo de funcionamento psíquico, a fim de auxiliar aqueles que trabalham com essa população. Utiliza como instrumentos a prova de Rorschach e a análise de entrevistas. Os sujeitos são 15 mulheres dependentes de álcool, encaminhadas por seus respectivos psiquiatras. Buscando elementos comuns, encontramos alterações significativas no modo de adaptação intelectual à realidade externa, marcado por estereotipias e submissão às imposições do meio, possivelmente decorrentes da necessidade de aprovação, ao mesmo tempo em que demonstram indiferença em relação ao outro, como se não sentissem afeto pelo outro e nem registrassem o afeto do outro, o que evidencia indiferenciação. O modo genérico e superficial de adaptação, a dispersão da fala, a turbulência afetiva, a não fixação e não retenção das experiências são aspectos que dificultam a criação e a inscrição de marcas significantes para além do "ser alcoolista". O universo fantasmático encontra-se presente, mas não integrado na dinâmica psíquica, havendo tendência a se negarem as próprias fantasias. O uso excessivo de álcool parece favorecer e manter a alienação do sujeito, na medida em que reforça o mecanismo de negação deixando as fantasias desalojadas psiquicamente. No que tange à sexualidade, pensamos o álcool como uma metonímia do homem ( $\varphi$ : álcool/garrafa/pênis/homem/pai) que desliza e se sustenta a partir de fantasias que trazem em seu cerne o significante devastação. Concluímos que a dependência de álcool pode eclodir na histeria, na neurose obsessiva e na psicose, sendo necessário um estudo mais aprofundado da função do uso de álcool em cada uma das estruturas psíquicas.

Palavras-chave: Alcoolismo. Mulheres. Constituição do sujeito. Psicodiagnóstico. Psicanálise. Teste de Rorschach. 


\begin{abstract}
MUNDURUCA, G. O. A contribution to the study of the psychic constitution of

alcoholic women. 2008, 204 f. Doctoral dissertation - Institute of Psychology, University of São Paulo, São Paulo, 2008.

This study examines the psychic constitution of alcoholic women and seeks to broaden the comprehension of their psychic functioning in order to assist those who work with this population. The study uses the Rorschach test and interview analysis as assessing instruments. The participants are 15 alcohol-dependent women who have been referred to us by their own psychiatrists. In search of common elements, we have found significant alterations in the intellectual mode of adaptation to external reality, characterized by stereotypies and submission to environmental impositions that probably derive from a need of approval. These characteristics also demonstrate indifference towards the other, as if the patients did not feel any affection for them and did not register the affection received from them. This fact displays undifferentiation. The generic and superficial adaptation mode, speech dispersion, affective turbulence, non-fixation and non-retention of experiences are aspects that hinder the creation and inscription of significant marks that go beyond the "alcoholic individual". Even though the phantasmic universe is present, it is not integrated into the psychic dynamics and the subjects present a tendency of denying their own fantasies. The excessive use of alcohol seems to favor and maintain the subject's alienation as the negation mechanism is reinforced and the fantasies are psychically displaced. As for sexuality, we think of alcohol as a metonymy for man ( $\varphi$ : alcohol/bottle/penis/man/father) with a flowing meaning that is supported by fantasies that carry the signifier devastation in their core. As a conclusion, we state that alcohol dependence may culminate in hysteria, obsessive neurosis and psychosis; however, a deeper study on the function of alcohol use in each of the psychic structures is still necessary.
\end{abstract}

Keywords: Alcoholism. Women. Constitution of the subject. Psychodiagnosis. Psychoanalysis. Rorschach test. 


\section{SUMÁRIO}

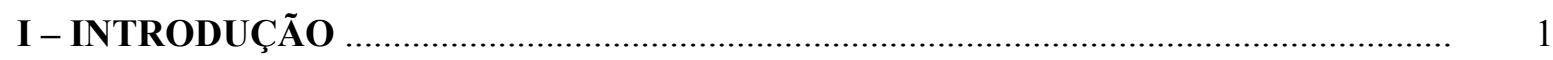

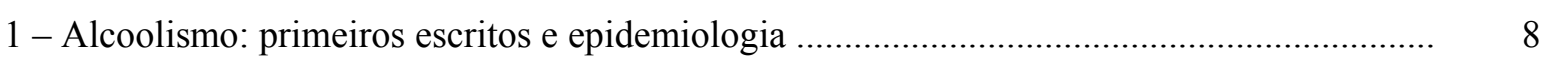

2 - O alcoolismo em mulheres: um panorama geral ................................................................... 15

3 - Alcoolismo feminino e comorbidade: algumas contribuições do pensamento psicanalítico e rorschachiano

4 - O alcoolismo pelo método de Rorschach ........................................................................ 44

5 - Alcoolismo feminino e sexualidade: o álcool como metonímia do homem para a mulher .. $\quad 50$

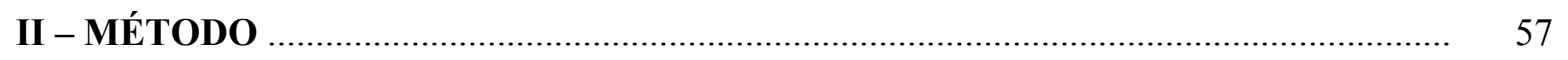

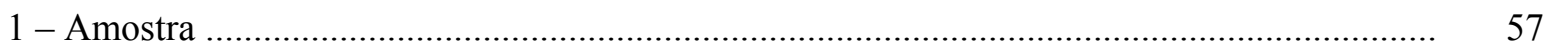

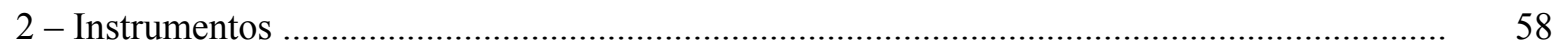

2.1 - A prova de Rorschach: características gerais ......................................................... 58

2.2 - Algumas considerações sobre a análise das entrevistas ............................................ $\quad 60$

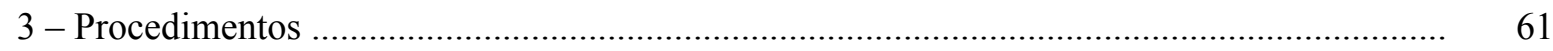

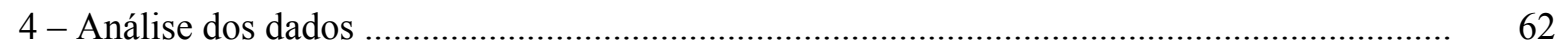

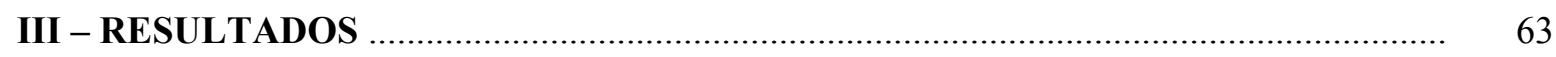

1 - Caracterização da amostra a partir de informações obtidas nas entrevistas ............................ 63

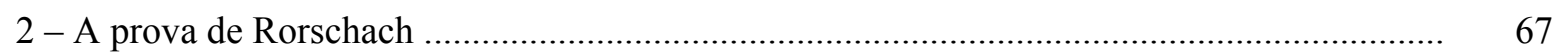

2.1 - Resultados quantitativos: uma apreciação estatística .................................................... 67

2.2 - Análise dos resultados quantitativos .................................................................... 73

3 - Apresentação dos casos a partir das alterações dos índices do Rorschach ........................... 86

$3.1-\mathrm{RMI} \uparrow[(\% \mathrm{~A}+\% \mathrm{~V}+\% \mathrm{~F}) \div 3]$ nas pranchas monocromáticas ................................. 86

$3.2-$ Conação $\downarrow\left[\right.$ Con $\left.=\% \mathrm{~F}^{+}-(100-\% \mathrm{~F})\right]$ e Lambda $\uparrow[\lambda=(\mathrm{R}-\mathrm{F}) \div \mathrm{F}]$ nas pranchas policromáticas

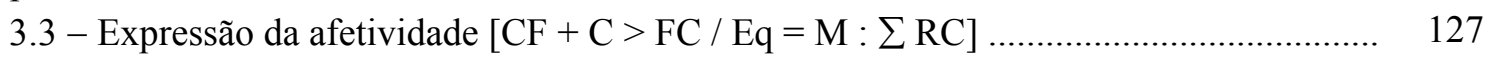

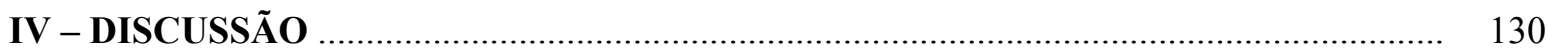

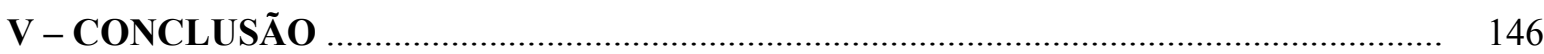

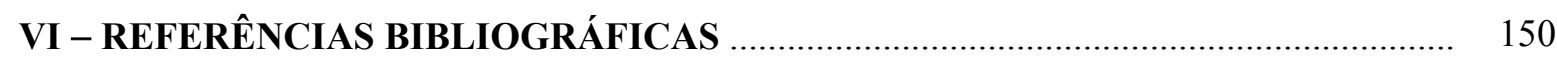

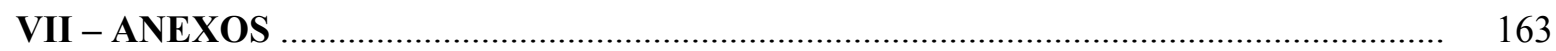

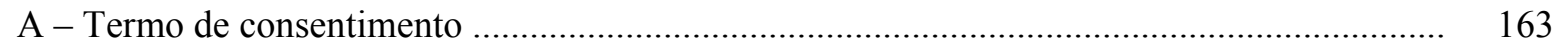

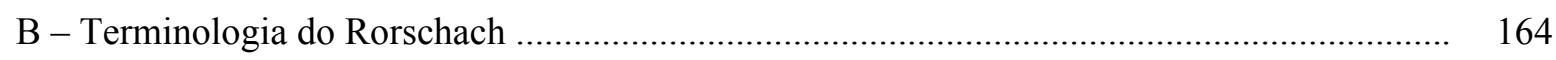

$\mathrm{C}$ - Ficha de fórmulas e expectativa em relação à população normal ........................................ 165

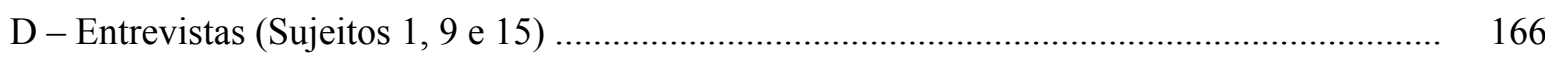

